\title{
Brain Abscess Sustained by Actinomyces meyeri in an Immunocompetent Patient
}

\author{
Gabriele Fabbri' ${ }^{1}$, Viola Guardigni ${ }^{1}$, Silvio Sarubbo ${ }^{2}$, Rosario Cultera ${ }^{1}$ and Carlo Contini ${ }^{1 *}$
}

${ }^{1}$ Department of Medical Sciences, Section of Infectious Diseases, University S. Anna Hospital, Italy

${ }^{2}$ Department of Neuroscience and Rehabilitation, Neurosurgery Unit, Azienda Ospedaliera-Universitaria, Arcispedale S. Anna, Italy

\begin{abstract}
Actinomycosis is a rare granulomatous disease, caused by filamentous Gram positive anaerobic bacteria, which may become pathogenic following disruption of anatomical barriers with invasion of host's deep tissues and dissemination of infection to any site of the organism. A. israelii is found in most clinical presentations.

We describe a case of a 41 years-old immunocompetent woman affected by thalamic abscess due to Actinomyces meyeri, presenting with fever, headache, vomit, confusion. A brain Computed Tomography (CT) and a Magnetic Resonance Imaging (MRI) revealed an expansive thalamic lesion with wide central necrosis and perilesionaledema. The patient was immediately addressed to a first evacuation of the mass, followed by a second drainage because of recurrence of the abscess. Microbiological and histological findings from intraoperatory specimens, resulted positive for $A$. meyeri. Ampicillin $20 \mathrm{~g}$ plus rifampicin $1200 \mathrm{mg} /$ day was started, followed by ceftriaxon $4 \mathrm{~g} / \mathrm{day}$ for 2 weeks and by amoxicillin $3 \mathrm{~g} /$ day. The treatment was discontinued after 9 months; brain MRls performed at the end of therapy and after 3 and 6 months from the interruption of therapy revealed a gradual but complete resolution of cerebral abscess.

Actinomycosis is an infrequent but severe disease that can mimic tuberculosis, nocardiosis and malignancies. Cases of cerebral actinomycosis reported in literature are limited, but those from $A$. meyeri are extremely rare. Moreover this pathogen is often related to disseminated disease, especially in immunocompromised patients. There is no consensus about treatment of cerebral actinomycosis: different types of regimens are proposed; we firstly administered ampicillin (12-16 g/day for at least six months), plus rifampicin, to enhance their penetration in CNS. Actinomycosis requires a prompt diagnosis and antibiotic treatment (often in combination with surgery) in order to avoid dissemination and prevent complications.
\end{abstract}

Keywords: Actinomycosis; Actinomyces meyeri; Brain; Abscess; Penicillin; Rifampicin

\section{Case and Methods}

Actinomycosis is a rare, chronic and slowly progressive granulomatous disease, caused by filamentous Gram positive anaerobic bacteria from Actinomycetaceae family (genus Actinomyces) belonging to endogenous flora of mucous membranes [1]. These bacteria normally colonize oral cavity, vagina and other mucous membranes but they become pathogenic and can initiate a prolonged chronic inflammatory process if the integrity of the mucosal barrier is compromised, with dissemination to any site of the organism [1].

Actinomycosis is classified into several clinical forms, according to anatomical site of infection: orocervicofacial, thoracic, abdominopelvic, central nervous system (CNS), musculoskeletal and disseminated forms. The clinical features are often misdiagnosed and anearly diagnosis needs a high level of clinical suspicion [2].

The involvement of CNS represents an uncommon condition (especially consequent to a haematogenous diffusion of microorganism), but it's undoubtedly the most critical site of infection. The disease usually is characterized by "mass effect" symptoms, such as headache, dizziness and focal neurological signs related to the site of involvement. Surgical approach is often necessary to improve medical therapy, especially for difficult localizations and large masses, with evidence of compression of nearest nervous structures.

In the last years, CNS localization by Actinomyces Spp. has been occasionally reported [3-6]: in particular abscesses by Actinomyces meyeri have been found in a small proportion of cases, especially in the context of disseminated diseases, with only secondary involvement of CNS [7].
We describe a young woman suffering from primary thalamic abscess due to A. meyeri, without any other systemic localization, successfully treated after prompt diagnosis and antibiotic therapy.

\section{Discussion}

A 41-years old woman was initially admitted to First Aid of our Hospital, because of fever $\left(38^{\circ} \mathrm{C}\right)$, headache, vomit, confusion and dysarthria. Emerging blood test showed mild leukocytosis (white blood cells $11840 / \mathrm{mmc}$, neutrophils $10510 / \mathrm{mmc}$ ), reactive-C protein (RCP) $7.50 \mathrm{mg} / \mathrm{dl}$, whereas a brain CT scan detected an expansive thalamic lesion with a wide central necrosis and perilesionaledema.

Her medical history was negative, apart from a dental fracture, treated with conservative approach six months before the onset of the clinical symptoms. Tuberculin sensitivity test, interferon-gamma releasing assay, HIV and Toxoplasma gondii serology were negative. Trans-thoracic echocardiography, chest X-ray, orthopantomogram

*Corresponding author: CarloContini, Department of Medical Sciences, Via Fossato di Mortara, 64b, University of Ferrara, I- Ferrara, Italy; Tel: +39 0532 239114/+39 0532 455490; Fax: +39 0532 455495; E-mail: cnc@unife.it

Received December 18, 2013; Accepted December 27, 2013; Published January 05,2014

Citation: Fabbri G, Guardigni V, Sarubbo S, Cultera R, Contini C (2014) Brain Abscess Sustained by Actinomyces meyeri in an Immunocompetent Patient. J Neurol Neurophysiol 5: 184. doi:10.4172/2155-9562.1000184

Copyright: ( 2014 Fabbri G, et al. This is an open-access article distributed under the terms of the Creative Commons Attribution License, which permits unrestricted use, distribution, and reproduction in any medium, provided the original author and source are credited. 
Citation: Fabbri G, Guardigni V, Sarubbo S, Cultera R, Contini C (2014) Brain Abscess Sustained by Actinomyces meyeri in an Immunocompetent Patient. J Neurol Neurophysiol 5: 184. doi:10.4172/2155-9562.1000184

and abdomen ultrasonography were negative. A brain MRI confirmed the mass, with a compressing effect over cerebral $3^{\text {rd }}$ ventricle (Figure 1). The patient was promptly addressed to Neurosurgery Department, in order to perform a diagnostic and therapeutic evacuation of the brain mass.

During surgical drainage, an intraoperatory specimen of the lesion was collected for microbiological tests and antibiotics consisting of ceftriaxone $4 \mathrm{~g} /$ day. i.v. associated to rifampicin $1200 \mathrm{mg} /$ day i.v, were administered. Results from microbiology detected A. meyeri (sensitive to all antibiotics tested by our reference laboratory). These were later confirmed by cytological and histological analyses (Figure 2).

A second brain MRI, repeated to define the outcome of neurosurgical procedure, showed recurrence of the abscess. Because of worsening of patient's conditions, a second evacuation was recommended; ceftriaxone was discontinued and ampicillin i.v. $(5 \mathrm{~g}$ every 6 hours/day) plus continuous infusion of vancomycin 2 g/day and rifampicin were given at the above dosage.

Three weeks after, the patient was addressed to Department of Infectious Diseases to continue antibiotic therapy. At the entry, the patient was febrile $\left(37.5^{\circ} \mathrm{C}\right)$, clinical conditions were quite fair and physical examination showed mild left lower limb hyposthenia. Blood examinations were normal. An electroencephalogram showed diffuse
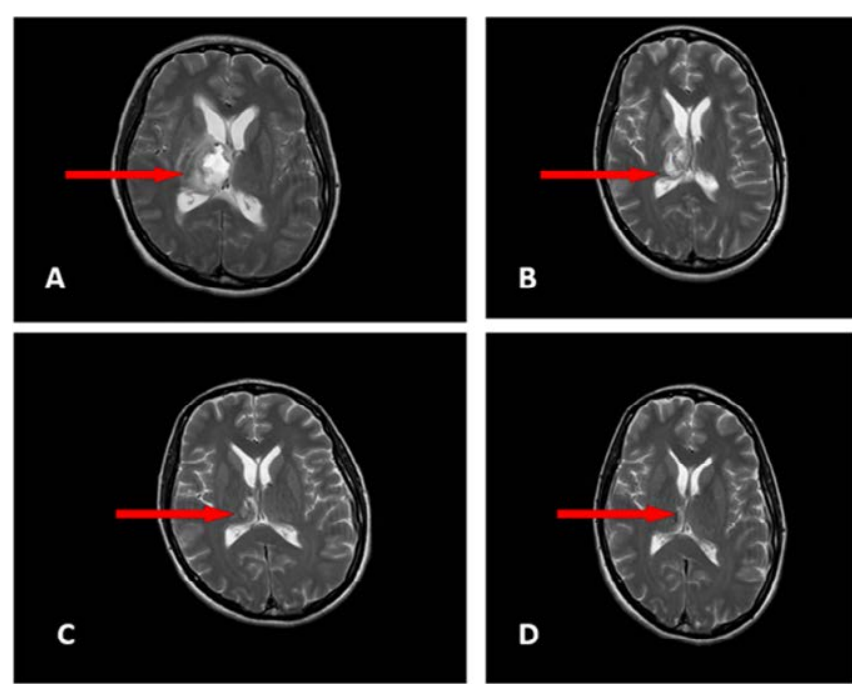

Figure 1: RM images showing evolution of actinomycotic brain abscess $(2.6 \times$ $2.5 \times 3.2 \mathrm{~cm}$ of diameter) at the time of diagnosis after 1 month, 3 and 6 months of therapy (Panel A, B, C and D respectively).
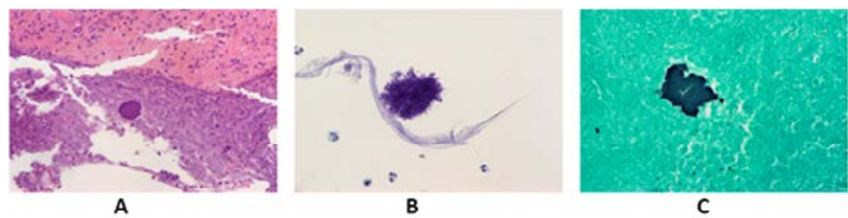

Figure 2: (A) Histological Examination of brain abscess revealing actinomycotic colony surrounded by necrosis and inflammatory infiltrate (hematoxylin and eosin stain, $\times 20$ ). (B) Photomicrograph of aspirate showing mixed inflammatory cell exudates and fluffy colonies. The actinomyces organisms radially arranged filamentous bacilli (hematoxylin and eosin stain, $\times 40$ ). (C) Cell block section showing positive silver impregnation of actinomycotic colonies(Grocott-Gomori methenamine-silver nitrate, $\times 40$ ). deceleration of electrical cortical activity. Antibiotic therapy was continued with only ampicillin 5 g every 6 hours in a day. Clinical conditions significantly improved with complete regression of fever and neurological symptoms. A further brain MRI did show significant reduction of abscess cavity and edema. The patient was therefore discharged with antibiotic treatment consisting of ceftriaxone, $4 \mathrm{~g} /$ day i.m. for 2 weeks, followed by amoxicillin $3 \mathrm{~g} /$ day, orally.

Admitted as outpatient to our Department after two weeks, the patient underwent brain MRIs at $3^{\text {rd }}$ and $6^{\text {th }}$ months of antibiotic treatment. These revealed a progressive resolution of cerebral abscess (Figure 1). Antibiotics were discontinued after 9 months, without any sign of recurrence of disease after 1 year of follow up.

\section{Conclusion}

Actynomicosis is an "endogenous infection". Among the more than 30 known species, $A$. israeli is the most common human pathogen. Less common species include $A$. naeslundii, A. odontolyticus, $A$. viscous, $A$. meyeri, $A$. turricensis and $A$. radingae.

The orocervical form includes about $50 \%$ of all reported cases [7]. It usually follows dental manipulation or trauma to the mouth. Other localizations consist of thoracic (15-20\%) and abdomino-pelvic (20\%) actinomycosis, whereas CNS is a rare site of disease and the infection typically arises from haematogenous spread or direct extension of orocervical infection. The disease usually occurs as brain abscess, meningitis/meningoencephalitis, actinomycoma, subdural empyema or epidural abscess [2].

A. meyeri can cause disseminated forms, by haematogenous spread and systemic involvement. In our case, CNS localization was primitive and no other site of infection was found. Moreover, A. meyeri was isolated from the brain. This was never described so far.

Reports of CNS actinomycosis by A. meyeri are extremely rare, especially in immunocompetent persons. One review described well documented cases of disseminated infection by A. meyeri, treated with drainage of infection focus and antibiotic therapy [8]. Other cases reported intracranial actinomycosis [3-6,9], but in none of them A. meyeri was recognized as the causative pathogen of primary brain abscess. Only one recent article [10] has reported a cervical actinomycosis with paravertebral abscess and spondylitis imputed to an infection by $A$. meyeri: in this case an avulsion of teeth 46 and 47 , presumed to be the entry point of the infectious process.

Our patient instead was immunocompetent, no predisposing factors for this infection were found and all causes of immunosuppression were excluded. Accumulating evidence suggests in fact that actynomicosis may be associated with impairment of immune system (transplantations, HIV, alcoholism, denutrition etc.) [11,12].

Despite the availability of various therapeutic options, the choice therapy seems to consist of high doses of beta-lactams, to which we firstly added rifampicin, in order to amplify the bactericidal effect and diffusion in the CNS. Although variable, the duration of therapy should be a long-term therapy, up to 12 months.

In our case, the early drainage of the abscess has allowed a relative short-term intravenous therapy, followed by a maintenance oral therapy, with good tolerability.

Because of the absence of predisposing factors, the diagnosis was difficult as actinomycosis can mimic tuberculosis, nocardiosis, other bacterial or mycotic abscesses and cancer. In this case, definitive 
Citation: Fabbri G, Guardigni V, Sarubbo S, Cultera R, Contini C (2014) Brain Abscess Sustained by Actinomyces meyeri in an Immunocompetent Patient. J Neurol Neurophysiol 5: 184. doi:10.4172/2155-9562.1000184

Page 3 of 3

diagnosis was reached and later confirmed by microbiological and histological features.

In conclusion, actinomycotic focal cerebral infections may occur with greater frequency than previously recognized. Although primary infection is extremely rare and controversial, A. meyeri should be always suspected even in immune competent patients with brain mass and/or neurological signs without evident risk factors.

\section{References}

1. Mandell GL, Bennet JE, Dolin R (2010) Principles and practice of Infectious Diseases. (7thedn), Elsevier Churchill Livingstone.

2. Wong VK, Turmezei TD, Weston VC (2011) Actinomycosis. BMJ 343: d6099.

3. Adeyemi OA, Gottardi-Littell N, Muro K, Kane K, Flaherty JP (2008) Multiple brain abscesses due to Actinomyces species. Clin Neurol Neurosurg 110: 847849.

4. Tsai MS, Tarn JJ, Liu KS, Chou YL, Shen CL (2001) Multiple actinomyces brain abscesses: case report. J Clin Neurosci 8: 183-186.

5. Puzzilli F, Salvati M, Ruggeri A, Raco A, Bristot R, et al. (1998) Intracranial actinomycosis in juvenile patients. Case report and review of the literature. Childs Nerv Syst 14: 463-466.
6. Ham HY, Jung S, Jung TY, Heo SH (2011) Cerebral actinomycosis: unusual clinical and radiological findings of an abscess. J Korean Neurosurg Soc 50: 147-150.

7. Apothéloz C, Regamey C (1996) Disseminated infection due to Actinomyces meyeri: case report and review. Clin Infect Dis 22: 621-625.

8. Weese WC, Smith IM (1975) A study of 57 cases of actinomycosis over a 36 -year period. A diagnostic 'failure' with good prognosis after treatment. Arch Intern Med 135: 1562-1568.

9. Akhaddar A, Elouennass, Baallal H, Boucetta M (2010) Focal intracranial infections due to Actinomyces species in immunocompetent patients: diagnostic and therapeutic challenges. World Neurosurg 74: 346-350.

10. Duvignaud A, Ribeiro E, Moynet D, Longy-Boursier M, MalvyD (2013) Cervica spondylitis and spinal abscess due to ActinomycesmeyeriBraz J Infect Dis, Nov 6. pii: S1413-8670(13)00239-0.

11. Murchan EM, Redelman-Sidi G, Patel M, Dimaio C, Seo SK (2010) Esophageal actinomycosis in a fifty-three-year-old man with HIV: case report and review of the literature. AIDS Patient Care STDS 24: 73-78.

12. Chaudhry SI, Greenspan JS (2000) Actinomycosis in HIV infection: a review of a rare complication. Int J STD AIDS 11: 349-355. 